\title{
Reseñas de Trabajos de Investigación en el Posgrado - PiEnCias
}

\author{
Reviews of Postgraduate Research Work - PiEnCias
}

\section{Maria Mercedes Jiménez Narváez*}

(iD) https://orcid.org/0000-0002-7402-4393

\author{
Tipo de Artículo: Reseñas
}

Doi: 10.17533/udea.unipluri.20.1.12

Cómo citar este artículo:

Jiménez Narváez, M. M. (2020). Reseñas de Trabajos de Investigación en el Posgrado - PiEnCias.

Uni-Pluriversidad, 20(1), e2020111. doi: 10.17533/udea.unipluri.20.1.12

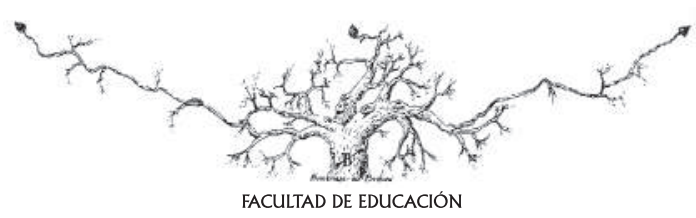

* Universidad de Antioquia. Colombia

Email: maria.jimenez@udea.edu.co 


\begin{tabular}{ll}
\hline $\begin{array}{l}\text { Nombres y apellidos } \\
\text { completos del autor/a: }\end{array}$ & Vanessa Arias Gil \\
\hline $\begin{array}{l}\text { Título del trabajo de } \\
\text { investigación: }\end{array}$ & $\begin{array}{l}\text { Las TIC en la educación en Ciencias en Colombia: una mirada a } \\
\text { la investigación en la línea en términos de su contribución a los } \\
\text { propósitos actuales de la educación científica. }\end{array}$ \\
\hline Año de finalización: & 2016 \\
\hline $\begin{array}{l}\text { Programa: } \\
\text { Maestría en Educación en Ciencias Naturales - Línea de }\end{array}$ \\
\hline $\begin{array}{l}\text { Enlace al trabajo } \\
\text { (CEDED): }\end{array}$ & http://ayura.udea.edu.co:8080/jspui/handle/123456789/2305 \\
\hline $\begin{array}{l}\text { Correo electrónico de } \\
\text { contacto: }\end{array}$ & vanessa.arias@udea.edu.co \\
\hline
\end{tabular}

Código ORCID: $\quad$ https://orcid.org/0000-0002-9449-6144

Perfil de Google Scholar: https://scholar.google.com/citations?hl=es\&user=HiaZiMwAAAAJ

\section{Reseña:}

La investigación partió del interés por conocer cómo se estaban incorporando las TIC en la enseñanza de las Ciencias en el ámbito colombiano, como insumo para favorecer su implementación en el aula con una fundamentación teórica que contribuya a aprender ciencias, a aprender a hacer ciencia y aprender sobre ciencia. En este sentido, se buscó responder a la pregunta ¿Cómo la producción científica en relación con la implementación de TIC en la Enseñanza de las Ciencias en Colombia contribuye con los retos actuales de la educación en este campo? Con respecto a la metodología, se optó por la investigación documental desde la perspectiva de Hoyos (2000). Esta metodología permitió un análisis de las publicaciones en las principales revistas de Educación en Ciencias a nivel nacional y de Educación y Educación en Ciencias a nivel internacional, teniendo como ventana temporal los años 2000 a 2014.
Para la base teórica se recurrió a Hodson (2003, 2010) y Moreira $(2005,2010)$, referentes que tienen en común una concepción de currículo y de Educación en Ciencias tendiente al desarrollo de un pensamiento crítico y una posición argumentada frente a la ciencia y su aprendizaje. Entre las principales conclusiones de la investigación se halla que la contribución de la producción científica en el campo de las TIC a los propósitos actuales de la Educación en Ciencias no se estaba dando en la medida esperada; que en las publicaciones relacionadas con TIC para la Enseñanza de las Ciencias, el principal interés seguía siendo la presentación de experiencias basadas en un uso instrumental de las tecnologías, y que la mayor carencia era la inclusión de elementos didácticos y epistemológicos. Finalmente, el estudio muestra que el estado de la investigación sobre este campo en Colombia, en comparación con el ámbito internacional, no presentaba grandes diferencias. 


\begin{tabular}{ll}
\hline $\begin{array}{l}\text { Nombres y apellidos } \\
\text { completos del autor/a: }\end{array}$ & Natalia Ramírez Agudelo \\
\hline $\begin{array}{l}\text { Título del trabajo de } \\
\text { investigación: }\end{array}$ & $\begin{array}{l}\text { El proceso de inserción profesional del profesor principiante de } \\
\text { Ciencias Naturales: cuatro casos en Colombia }\end{array}$ \\
\hline Año de finalización: & 2016 \\
\hline Programa: & $\begin{array}{l}\text { Maestría en Educación en Ciencias Naturales - Línea formación } \\
\text { de profesores }\end{array}$ \\
\hline \begin{tabular}{l} 
Enlace al trabajo (CEDED): \\
http://hdl.handle.net/10495/5283 \\
\hline contacto:
\end{tabular}$\quad$ natalia.ramirez2@udea.edu.co \\
\hline Código ORCID: & https://orcid.org/0000-0001-6301-5187 \\
\hline Perfil de Google Scholar: & $\begin{array}{l}\text { https://scholar.google.com/ } \\
\text { citations?user=mleJUjoAAAAJ\&hl=es }\end{array}$ \\
\hline
\end{tabular}

\section{Reseña:}

La inserción profesional hace referencia al momento en el cual el profesor inicia el ejercicio de la docencia, posterior a su formación inicial. Esta etapa es relevante en tanto posee características que podrían determinar la trayectoria profesional de cada docente, y refiere problemas y aprendizajes particulares que constituyen ese tránsito de estudiante a docente. El proyecto empleó la metodología de investigación cualitativa con un enfoque de estudio de caso colectivo y contó con la participación de 24 profesores principiantes de licenciaturas afines a las Ciencias Naturales, egresados de la Universidad de Antioquia, la Universidad Industrial de Santander, la Universidad del Tolima y la Universidad Surcolombiana. Esta metodología posibilitó la comprensión de su proceso de inserción profesional desde cuatro contextos diferentes. La recolección de información buscó comprender las ex- periencias de los participantes; su situación laboral y las rutinas empleadas para adquirir su primer empleo; y caracterizar qué problemas presentaban para analizarlos desde los niveles de socialización propuestos por Jordel (1987), desde lo personal, las situaciones de aula o clase, lo institucional y lo social.

Se encontró que los saberes disciplinares no constituyeron un problema para los participantes, el problema recaía en el manejo de grupo y en la falta de acompañamiento por parte de la Institución Educativa donde laboraban. Tomando en cuenta que la inserción es "un proceso de inicio al mercado laboral, a una institución y a una organización” (Tardif y Borges, 2013, p. 29), se concluye que la obtención del empleo y la adaptación son elementos importantes en el inicio del ejercicio de la profesión $\mathrm{y}$, dependiendo de éstos, ese ingreso puede ser difícil o fácil. 


\begin{tabular}{ll}
\hline $\begin{array}{l}\text { Nombres y apellidos } \\
\text { completos del autor/a: }\end{array}$ & Yesenia Quiceno Serna \\
\hline $\begin{array}{l}\text { Título del trabajo de } \\
\text { investigación: }\end{array}$ & $\begin{array}{l}\text { (Re)constitución de la identidad profesional del profesor } \\
\text { principiante de Ciencias: una visión desde sus saberes en la etapa } \\
\text { de inserción a la docencia }\end{array}$ \\
\hline Año de finalización: & 2016 \\
\hline Programa: & $\begin{array}{l}\text { Maestría en Educación en Ciencias Naturales -Línea formación } \\
\text { de profesores }\end{array}$ \\
\hline $\begin{array}{l}\text { Correo electrónico de } \\
\text { contacto: }\end{array}$ & yesenia.quiceno@udea.edu.co, yesenia.qserna@gmail.com \\
\hline Código ORCID: & https://orcid.org/0000-0002-7735-4208 \\
\hline Perfil de Google Scholar: & $\begin{array}{l}\text { https://scholar.google.com/ } \\
\text { citations?user=Rdr3QJQAAAAJ\&hl=es\&authuser=1 }\end{array}$ \\
\hline
\end{tabular}

\section{Reseña:}

La investigación indagó ¿Cómo se (re) constituye la identidad del profesor principiante de Ciencias Naturales a partir de los saberes profesionales presentes en la inserción a la docencia? Como propósito, situó la descripción de los imaginarios profesionales de los profesores principiantes y las condiciones reales del ejercicio docente. Para este fin, se identificaron los saberes que moviliza el profesor de Ciencias en su paso de estudiante dirigido a profesional autónomo, caracterizando las fuentes de donde se sustentan y su relación con la identidad profesional. La investigación se enmarcó en un paradigma cualitativo-interpretativo desde un enfoque biográfico-narrativo; se realizaron entrevistas, autobiografías y un grupo de discusión final. Se cruzaron las historias de vida de cinco profesores principiantes de ciencias, egresados del mismo programa de formación inicial, con la intención de construir de manera conjunta, un relato polifónico que diera cuenta de las relaciones entre los saberes, la cultura profesional y la identidad docente en la inserción profesional.

Como conclusiones, se resalta la importancia de los contextos de inserción y las posibilidades que brindan a los jóvenes profesores para su desarrollo profesional, impactando positiva o negativamente su permanencia y compromiso con el trabajo. Así, el contexto de llegada realiza un modelado de los constituyentes de la identidad, que se van transformando o no, de acuerdo con las experiencias que los profesores adquieren en el ejercicio de su profesión.

Finalmente, se indica que comprender la (re)constitución identitaria de los profesores lleva a reconocer cómo las representaciones construidas sobre la profesión, el trabajo y la función docente se consolidan durante la formación escolar e inicial. El inicio en la docencia es un momento de validación y de confrontación -más que de construcción-, donde el profesor principiante va acoplándose paulatinamente a los desafíos de la actividad profesional. 


\begin{tabular}{ll}
\hline $\begin{array}{l}\text { Nombres y apellidos } \\
\text { completos del autor/a: }\end{array}$ & Mónica Eliana Cardona Zapata \\
\hline $\begin{array}{l}\text { Título del trabajo de } \\
\text { investigación: }\end{array}$ & $\begin{array}{l}\text { La actividad experimental apoyada en el uso de sistemas de } \\
\text { adquisición de datos: una propuesta teórico-metodológica para } \\
\text { favorecer la conceptualización en Física. }\end{array}$ \\
\hline Año de finalización: & 2018 \\
\hline Programa: & $\begin{array}{l}\text { Maestría en Educación en Ciencias Naturales - Línea de } \\
\text { investigación en TIC para la Enseñanza de las Ciencias }\end{array}$ \\
\hline Enlace al trabajo (CEDED): & http://ayura.udea.edu.co:8080/jspui/handle/123456789/3221 \\
\hline $\begin{array}{l}\text { Correo electrónico de } \\
\text { contacto: }\end{array}$ & meliana.cardona@udea.edu.co \\
\hline Código ORCID: & https://orcid.org/0000-0002-3697-2288 \\
\hline Perfil de Google Scholar: & $\begin{array}{l}\text { https://scholar.google.es/ } \\
\text { citations?user=v80NzkAAAAJ\&hl=es\&oi=ao }\end{array}$ \\
\hline
\end{tabular}

\section{Reseña:}

Esta investigación tuvo como propósito estudiar la contribución de los Sistemas de Adquisición de Datos (SAD) a la conceptualización en Física, a partir de la implementación de una propuesta didáctica fundamenta en la Teoría de los Campos Conceptuales de Vergnaud (1990). Este autor enfatiza en la conceptualización a partir de situaciones que ponen en juego diversos componentes de la estructura cognitiva de los estudiantes. En coherencia con este referente, se diseñó un diagrama heurístico llamado Adaptación de la V de Gowin para el uso de SAD (AVSAD), para identificar el desarrollo cognitivo de los estudiantes al enfrentarse a situaciones experimentales novedosas en el trabajo de laboratorio en Física; asimismo, el diseño estuvo basado en el modelo de aprendizaje MATLaF de Andrés y Pesa (2004).

La investigación se enmarcó en el paradigma cualitativo y se llevó a cabo con profesores en formación de un programa de Licenciatura en Ciencias Naturales de la
Universidad de Antioquia. La valoración de la contribución de los SAD, junto con los elementos teóricos y metodológicos, responde a una preocupación por resignificar la actividad experimental para la formación de profesores de Ciencias y de Física en particular. Los resultados permitieron concluir que (1) el uso de SAD posibilitó a los estudiantes identificar relaciones entre variables, representar los fenómenos involucrando diferentes sentidos y mejorar la comprensión de los conceptos; (2) a través del AVSAD se fortaleció el carácter complementario de la relación teoría - práctica y el análisis de la estructura de un experimento; (3) el uso de $\mathrm{SAD}$ en la formación de profesores requiere criterios pedagógicos y didácticos que permitan valorar constantemente la conceptualización de los estudiantes y su progresividad en el acercamiento a la construcción del conocimiento científico, siendo coherentes con las necesidades e intereses de la educación actual. 


\begin{tabular}{|c|c|}
\hline $\begin{array}{l}\text { Nombres y apellidos } \\
\text { completos del autor/a: }\end{array}$ & Adriana María Villegas Otálvaro \\
\hline $\begin{array}{l}\text { Título del trabajo de } \\
\text { investigación: }\end{array}$ & $\begin{array}{l}\text { Aportes de la práctica pedagógica a la constitución de la } \\
\text { identidad profesional de los maestros en formación inicial de } \\
\text { Ciencias Naturales }\end{array}$ \\
\hline Año de finalización: & 2018 \\
\hline Programa: & $\begin{array}{l}\text { Maestría en Educación en Ciencias Naturales. Línea formación } \\
\text { de profesores }\end{array}$ \\
\hline Enlace al trabajo (CEDED): & http://200.24.17.68:8080/jspui/handle/123456789/3438 \\
\hline $\begin{array}{l}\text { Correo electrónico de } \\
\text { contacto: }\end{array}$ & adrimvillegas@gmail.com \\
\hline Código ORCID: & https://orcid.org/0000-0003-2563-2496 \\
\hline Perfil de Google Scholar: & $\begin{array}{l}\text { https://scholar.google.com/citations? user }=\text { tOjfd0AAAAJ\&hl }=\mathrm{e} \\
\text { s\&citsig=AMD79oq74V-gltPxpPK0N3dF7gAqWuOsA }\end{array}$ \\
\hline
\end{tabular}

\section{Reseña:}

En las últimas décadas, la línea de investigación sobre profesores de Ciencias Naturales se ha consolidado como campo de interés y potencialidad en el contexto educativo. En este sentido, durante los años 2016 y 2018, se desarrolló una investigación que buscó analizar los aportes que brinda la práctica pedagógica a la constitución de la identidad profesional de los maestros en formación inicial de esta área del conocimiento. El estudio se realizó mediante el enfoque biográfico-narrativo y los participantes fueron cinco maestros en formación que se realizaban su práctica pedagógica en Instituciones Educativas de la ciudad de Medellín.

Una vez se finalizó la investigación, se concluyó que esta aportó no solo a la identificación de elementos reflexivos sobre el papel de la práctica en la constitución de la identidad de los futuros profesores y a la forma de ver la profesión docente, sino a los procesos formativos que se llevan en la actualidad en el programa y que permitieron proponer algunas recomendaciones para mejorar la formación de los futuros maestros de Ciencias. 


\begin{tabular}{|c|c|}
\hline $\begin{array}{l}\text { Nombres y apellidos } \\
\text { completos del autor/a: }\end{array}$ & $\begin{array}{l}\text { Janes Jahir García Montoya y Johan Camilo Fernández } \\
\text { Bedoya }\end{array}$ \\
\hline $\begin{array}{l}\text { Título del trabajo de } \\
\text { investigación: }\end{array}$ & $\begin{array}{l}\text { La enseñanza del autocuidado como estrategia fundamental en la } \\
\text { promoción de la salud }\end{array}$ \\
\hline Año de finalización: & 2020 \\
\hline Programa: & Maestría en Educación Modalidad Virtual \\
\hline Enlace al trabajo (CEDED): & $\begin{array}{l}\text { http://bibliotecadigital.udea.edu.co/ } \\
\text { bitstream/10495/13943/1/FernandezBedoyaJoan_2020_ } \\
\text { Ense\%C3\%B1anzaAutocuidadoPromocion.pdf }\end{array}$ \\
\hline $\begin{array}{l}\text { Correo electrónico de } \\
\text { contacto: }\end{array}$ & janes.garcia@udea.edu.co,johan.fernandez@udea.edu.co \\
\hline \multirow[t]{2}{*}{ Código ORCID: } & https://orcid.org/0000-0002-2345-2291 \\
\hline & https://orcid.org/0000-0002-8534-1319 \\
\hline \multirow[t]{2}{*}{ Perfil de Google Scholar: } & $\begin{array}{l}\text { https://scholar.google.com/citations?view_op=list_ } \\
\text { works\&hl=es\&user=9VY2uXoAAAAJ }\end{array}$ \\
\hline & $\begin{array}{l}\text { https://scholar.google.es/citations?view_op=list } \\
\text { works\&hl=es\&authuser=2\&user=azbpwtgAAAAJ }\end{array}$ \\
\hline
\end{tabular}

\section{Reseña:}

La investigación indaga sobre cómo la enseñanza del autocuidado aporta a la promoción de la salud de los estudiantes de los grados octavo y noveno de dos instituciones educativas del departamento de Antioquia (Colombia). Este estudio se realizó bajo el método de Investigación Acción Educativa y recolectó información a través de técnicas narrativas como grupo focal, entrevista, encuesta y diario de campo, que permitieron reconocer las concepciones de los estudiantes sobre el autocuidado y sus prácticas de salud. La información se usó para la construcción de cinco secuencias didácticas, cuya aplicación permitió abrir un espacio en el aula donde la enseñanza del autocuidado fue pensada y planeada a partir de las nece- sidades de los estudiantes de las dos instituciones educativas. Posterior a la aplicación de las secuencias didácticas, se valoró cómo este proceso de enseñanza del autocuidado aportó a la promoción de la salud de los estudiantes. Los resultados evidenciaron que, en algunos contenidos relacionados con la salud, los estudiantes demostraron ser más conscientes en temas como alimentación, higiene personal y consumo de sustancias psicoactivas. En las dos instituciones donde se implementó el proyecto se pudo observar que los estudiantes se interesaban y motivaban por tratar asuntos que afectaban directamente su salud. 


\begin{tabular}{ll}
\hline $\begin{array}{l}\text { Nombres y apellidos } \\
\text { completos del autor/a: }\end{array}$ & Jhon Daniel Pabón Rúa \\
\hline $\begin{array}{l}\text { Título del trabajo de } \\
\text { investigación: }\end{array}$ & $\begin{array}{l}\text { El trabajo práctico de laboratorio y el papel de los instrumentos } \\
\text { tecnológicos en la enseñanza de la Física. }\end{array}$ \\
\hline Año de finalización: & 2020 \\
\hline Programa: & Maestría en Educación en Ciencias Naturales \\
\hline Enlace al trabajo (CEDED): & $\begin{array}{l}\text { http://bibliotecadigital.udea.edu.co/bitstream/10495/15044/1/ } \\
\text { PabonJhon_2020_LaboratorioEnsenanzafisica.pdf }\end{array}$ \\
\hline $\begin{array}{l}\text { Correo electrónico de } \\
\text { contacto: }\end{array}$ & jhon.pabon@udea.edu.co \\
\hline Código ORCID: & https://orcid.org/0000-0001-5050-4125 \\
\hline Perfil de Google Scholar: & $\begin{array}{l}\text { https://scholar.google.com/citations?view_op=list_ } \\
\text { works\&hl=es\&user=LNO9JVwAAAAJ }\end{array}$ \\
\hline
\end{tabular}

\section{Reseña:}

La literatura reporta que en los trabajos experimentales de Física se presenta una clara división entre teoría y práctica que da cuenta de una visión distorsionada de la construcción del conocimiento científico. Además, esta visión ha hecho que se vea desfavorecido el desarrollo de procesos cognitivos de orden superior de los sujetos, por limitar este espacio de aprendizaje al seguimiento de guías pautadas con objetivos preestablecidos. En este sentido, se construyó una propuesta teórico-metodológica fundamentada en la Teoría de los Campos Conceptuales de Gerard Vergnaud, en la que se articulaban los trabajos prácticos de laboratorio y el uso de tecnologías para abordar situaciones que favorecieran la conceptualización en Física. La propuesta se implementó con un grupo de maestros en formación de la Licenciatura en Ciencias Naturales de la Universidad de Antioquia, con quienes se llevó a cabo un estudio de caso desde una perspectiva cualitativa. Se observó la evolución de los esquemas de los participantes a partir del enriquecimiento de sus invariantes operatorios y la generación de secuencias de acción pertinentes para las situaciones a las que se enfrentaban. Por otra parte, a la luz de la Teoría Sociocultural de Vygotsky, se encontró que los sistemas de adquisición de datos se posicionaban como auténticos instrumentos de mediación cultural, tanto desde un punto de vista simbólico como técnico. Además, estos dispositivos les permitieron transformar el entorno en función de los objetivos subyacentes a las situaciones con las que se enfrentaban. Se afirma que la implementación de la propuesta en cursos de formación inicial y continuada de profesores de ciencias puede contribuir a la conceptualización en Física, a la concepción del trabajo experimental como un espacio en el que confluyen teoría y práctica y a la apropiación crítica del uso de tecnologías. 